\title{
Aportaciones político-educativas de las Escuelas de Segunda Oportunidad (E2C) en Europa. El éxito del fracaso
}

García Redondo, Eva

Facultad de Educación- Universidad de Salamanca, Salamanca, España

evagr@usal.es

González López, Víctor

Facultad de Educación- Universidad de Salamanca, Salamanca, España vgl@usal.es

Revesado Carballares, David

Facultad de Educación- Universidad de Salamanca, Salamanca, España drevesado@usal.es

\section{Resumen}

En las últimas décadas, estamos comprobando cómo se está produciendo un constante flujo de iniciativas pedagógicas que pretenden poner solución a la exclusión social y económica de los más jóvenes. Sin duda, es la iniciativa europea Second Chance Schools (E2C), que cumple en estas fechas 20 años de aplicación, la que de manera más firme, organizada e institucionalizada, se ha configurado como imprescindible para alcanzar la igualdad de oportunidades desde la Escuela.

Valiéndonos del método de revisión bibliográfica, en combinación con el enfoque sociocrítico, pretendemos conocer cuáles son los orígenes de esta propuesta, que anticipamos a mediados de los años 90 , cuál es su evolución político-educativa así como, finalmente, el reflejo que esta presenta, en la actualidad, en los distintos países de Europa. Conseguiremos, de este modo, descubrir, por un lado, si la E2C puede y debe ser considerada como una acción de carácter supranacional y, por otro, si esta es un garante de equidad educativa capaz de alcanzar éxito a partir del fracaso. Para ello, remitimos una discusión en la que ponemos sobre el papel aquellos pros y contras, ventajas y desventajas, de esta apuesta socioeducativa tan alabada como criticada, concluyendo, finalmente, con un planteamiento novedoso respecto a las potencialidades y posibilidades que, aún poco reconocidas u ocultas, creemos que tienen estas escuelas de la oportunidad.

\section{Abstract}

Over the last decades, we have confirmed how a constant flow of pedagogical initiatives have tried to solve the social and economic exclusion of the youngest. Definitely, the European initiative "Second Chance Schools" (E2C), which is celebrating its 20 years of application, has become essential to achieve equal opportunities from the School, thanks to its firmness, organization and institutionalization.

Our proposal includes a bibliographic review in combination with a socio-critical approach. We intend to know what its origins are -which we anticipated in the mid-90s-, what its political- educational evolution is as well as the reflection it currently presents in the different countries of Europe. In this way, on the one hand, we will discover whether the E2C can and should be considered as a «supranational action» and, on the other hand, whether it is an educational equity guarantor essential to achieve success after failure. To this end, we are expounding a discussion in which we put on paper the pros and cons, advantages and disadvantages, of this socio- educational bet, as praised as criticized, concluding, finally, with a novel approach regarding the potentialities and possibilities that, still little recognized or hidden, we believe that these «opportunity schools'» have.

Palabras clave: Escuelas de Segunda Oportunidad, equidad educativa, igualdad de oportunidades, exclusión, Europa.

Keywords: Second Chance Schools (E2C), educational equity, equal opportunity, exclusion, Europe.

\section{INTRODUCCIÓN}

Es bien sabida la importancia que la educación tiene en la construcción de la persona entendida como ser social, profesional y cultural, sin embargo y pese a ello, siguen siendo contadas las iniciativas que atienden las singularidades de los estudiantes que no alcanzan los patrones de rendimiento socioeducativo considerados como aceptables para la promoción escolar. 
Las Escuelas de Segunda Oportunidad, traducción al castellano que ha tomado la propuesta europea Second Chance Schools (E2C), nacieron en 1995 con la idea de «promover la inserción social y educativa de un alumnado en general muy vulnerable y en evidente riesgo de exclusión» (Corchuelo, Cejudo, González-Faraco, Morón, 2016, p. 95). En otras palabras, las E2C tratan de fomentar, a través de sus políticas, la igualdad de oportunidades.

El «Libro blanco sobre la educación y la formación, Enseñar y aprender», publicado por la Comisión Europea (1996), reconoce varios de los problemas más acuciantes del espectro educativo. La desigualdad, la exclusión y la falta de referentes sociales asumen un punto de partida cada vez menos excepcional y, por ende, más habitual y cotidiano. La preocupación en Europa radica en hacer frente a un panorama que resulta desolador, más aun cuando centramos nuestra atención en España, uno de los países de la Unión con mayor tasa de fracaso escolar, aunque no solo de manera exclusiva, puesto que encontramos ejemplos en Alemania, donde su esfuerzos van «from motivation driven by structure and content, through to financial incentives, increase in prestige and a more attractive programme» (European Commission, 2018) o en Francia, donde la constitución de la Association Nacionale des Ėcoles de la 2e Chance ha supuesto un impulso imprescindible para los entre 100.000 y 130.000 alumnos desahuciados del sistema educativo, «providing them with a pathway to employment, combining professional and social support» (European Commission, 2018a).

Sea como fuere y adoptando diversas formas, el fomento y la garantía educativa es un derecho reconocido en todos los países de Europa, que supone, en la práctica, un compromiso incumplido y restringido, en ciertos sentidos, por falta de opciones válidas para su consecución. De todo ello intentaremos dar cuenta en las próximas líneas, mostrando qué son las escuelas de Segunda Oportunidad en Europa, quiénes son sus destinatarios, directos e indirectos, qué modelo pedagógico y fundamentos asume esta propuesta y, finalmente, sobre qué propuestas futuras se ha avanzar a nivel político educativo para construir un formato de escuela oportuna.

\section{QUÉ SON LAS ESCUELAS DE SEGUNDA OPORTUNIDAD}

Como anticipábamos, el panorama educativo europeo se ha visto modificado, en las últimas tres décadas, con el fin principal de dar opción a la inclusión de un alto porcentaje de estudiantes que no encontraban encaje en los modelos tradicionales de Escuela. Desde 1995, el interés de las políticas socioeducativas de la Unión Europea se ha centrado en la conformación de modelos más flexibles y ciertamente proactivos. Como bien apunta García (2005) las escuelas reactivas, que siguen siendo una rémora de nuestra cultura y tradición, están dando paso, progresivo y firme, hacia modelos que favorecen formas de trabajo y experiencias mucho más amplias, consecuentes y sociales. Las escuelas proactivas se encuentran, por el momento y de manera mayoritaria, fuera del sistema formal y suponen ejemplos que remiten alta capacidad adaptativa y de permeabilidad socioeducativa. La innovación, la porosidad y la prioridad individual superan los modelos tradicionales de una única escuela, con una exclusiva metodología y unos contenidos estancos. Estamos, por tanto, asumiendo un cambio de paradigma de escuelas reactivas, donde prima la meritocracia, la homogeneidad y el centralismo (Marchesi, 2003) hacia escuelas proactivas, donde se fomenta lo individual, la heterogeneidad y la diversidad.

Las Escuelas de Segunda Oportunidad son, por tanto, espacios educativos encargados de «desarrollar programas específicos para atender a la población que no lograba terminar su escolarización con éxito y quedaba excluida económica y socialmente, generando al Estado importantes gastos en subsidios sociales» (Corchuelo y Cejudo, 2013, sp.). De esta definición se extraen dos importantes hechos. El primero el que tiene relación con la esencia de las propias escuelas en cuanto que reflejan autenticas practicas pedagógicas donde prima el quién y no el cuánto y «revelan una atención cuidada a planes de aprendizaje personalizados; cultivo de habilidades de empleo, de habilidades para la vida y participación en experiencias de trabajo como parte integrante de la oferta formativa; reconocimiento del potencial de aprendizaje de actividades de distinta naturaleza, no sólo las académicas; y, en general, la importancia de desarrollar enfoques de intervención integral y holística» (González y San Fabian, 2018, p. 53). El segundo, que son instituciones que asumen responsabilidad política por una cierta "obligación» de defensa del sistema social público y de la igualdad de oportunidades. Es, en este sentido, en el que Fernández (s.f), citando a Osten (2000), destaca algunos de los rasgos que componen estas Escuelas y que se refieren a:

1. Ser considerados como proyectos con una amplia dimensión europea, lo que supone un carácter transnacional, flexible e integrado.

2. Regirse por los protocolos y directrices que marca la Comisión. 
3. Autoconstituirse como espacios con una gestión local y regional.

4. Participar de manera activa en la propia organización y sostenimiento, estimando que el éxito o fracaso de las mismas depende del trabajo diario de las mismas.

5. Favorecer una metodología alejada de convencionalismos y caracterizada por la atención a las necesidades en un orden prioritario y ajustado a las expectativas.

6. Responder a patrones educativos asentados sobre la construcción de un aprendizaje permanente en los ámbitos formal, no formal e informal.

7. Fomentar las relaciones entre diversas instituciones con las que se comparte fines, estrechando lazos, realizando aportaciones, compartiendo.

8. y, finalmente, evitar que se conviertan en "ghettos», optando más por la idea de alternativa real, factible y aceptada a nivel social y político-educativo.

Sin embargo, destacamos, en este punto, el caso de Finlandia que, aun siendo considerado como uno de los mejores países europeos y mundiales a nivel educativo (así lo indican los resultados de los últimos informes PISA), mantiene la no necesidad de escuelas de segunda oportunidad segregadas de las habituales dirigidas a adultos. Ello nos da muestra de la gran diversidad de problemática existente en Europa en lo que a fracaso escolar se refiere, mostrándonos realidades altamente diversas.

\section{DESTINATARIOS DIRECTOS E INDIRECTOS.}

Aquellos países que han establecidos Escuelas de Segunda Oportunidad reconocen beneficiarios que no son sino aquellos que más lo necesitan. Hablamos de jóvenes, hasta los 25 años, que no encuentran en la escuela reflejo de su cultura (Prieto, 2015) y que, por diversas causas que explicaremos a continuación, se han visto excluidos de un sistema único y cerrado de educación.

La Escuela legitima una cultura, la de la mayoría, que en un gran número de ocasiones no responde a los valores, principios y necesidades de la totalidad. Siendo así, esta reproduce un modelo único de pensamiento, sentimiento y evolución que remite confrontaciones ciertamente evitables. Es, en este objetivo, en el que se centran estas Escuelas entendidas para aquellos estudiantes que necesitan de un acompañamiento distinto al de la mayoría, alumnos que, según indica San Fabián (1999), sufren una discontinuidad pedagógica entre etapas educativas.

Centrándonos en los destinatarios directos y al hilo de lo anterior, conviene reseñar que son muchas las características que estos jóvenes presentan de manera común. Tal y como afirman Côté y Bynner (2008), «la exclusión social tiene sus antecedentes en una vida doméstica desfavorecida, la falta de apoyo de los padres a la educación y el rendimiento continuo deficiente a través del sistema educativo» (p.255). Por tanto, problemas familiares, comportamentales, fracasos educativos continuados, marcados por el absentismo e incluso el abandono, inmadurez, falta de alternativas y posibilidades laborales, ausencia de apoyo público así como de capacidad para resolver conflictos, propios y ajenos (Wilkinson y Pickett, 2010), son solo algunas de ellas. Por su parte, los destinatarios indirectos, sociedad y familia, entendemos que se benefician de estas iniciativas pedagógicas en tanto que conforman espacios aprovechable no solo para los jóvenes sino para el desarrollo cívico de la comunidad. En este sentido, se torna imprescindible el trabajo de las E2C como agentes dinamizadores del cambio político, lo que propone Echeita (2013) como proyecto exigente y exigible que permita alcanzar el desarrollo de todas y cada una de las capacidades y ritmos de los talentos excluidos (Corchuelo, Cejudo, González-Faraco, Morón, 2016).

\section{MODELO PEDAGÓGICO Y FUNDAMENTOS DE LAS E2C}

El modelo pedagógico que defienden estas escuelas está basado en la búsqueda constante del éxito social fuera de los entornos escolares clásicos y formalizados. Así lo defienden Gasch y Navas (2017) puntualizando que estas instituciones han de centrarse en atajar la vulnerabilidad que padecen sus estudiantes. Esta misma idea es compartida por Olmos y Mas (2013) quienes añaden la idea de trabajar por alcanzar una participación efectiva inserta en auténticas comunidades. Para ello, resulta 
imprescindible fundamentar su programa educativo sobre la idea ya clásica de que el trabajo es un derecho social y una forma de participación social (Wehmeyer, 1992), pese a reconocer la escasa oportunidad que, a día de hoy, ofrece el propio sistema laboral.

Siendo así, las E2C proponen un sistema educativo fundamentado en la flexibilidad, la práctica y el conocimiento técnico, elementos todos ellos que contribuyen a la construcción sociolaboral y personal de todos los individuos. Esta idea concuerda fielmente con la defendida en los años 90 por Delors (1996) y que alude a la necesidad de un aprendizaje permanente que reporte competencias en lo técnico y en lo práctico. No hablan ni defienden la idea clásica de meritocracia, ni tan siquiera la tendencia actual hacia la vulgarmente llamada «titulitis», sino la defensa de un programa que defienda la comunicación, la capacitación y el saber hacer, en todo momento y lugar, como elementos claves para la construcción de una nueva ciudadanía que contribuya al buen funcionamiento de los Estados (Corchuelo y Cejudo, 2015).

La orientación sociolaboral y socioeducativa es, en este sentido que venimos comentando, imprescindible a la vez que irrenunciable para la construcción de auténticos, genuinos y justos proyectos de vida. Es, por ello, por lo que sus planes de estudios giran en torno a la adquisición de competencias clave y habilidades laborales, ofreciendo a todo estudiante disfrutar de contextos educativos, sociales y laborales fiables, ricos y abiertos, permitiéndoles, por ende, el acceso a un empleo con un mínimo grado de cualificación. Ello requiere ofrecer a los alumnos, no solo conocimientos estancos y caducos, sino actitudes y habilidades prácticas, consecuentes con sus motivaciones, emociones y expectativas (Tiana, Moya y Luengo, 2011). Sin embargo, no todo es tan sencillo ni depende solo de las posibilidades abiertas por estos programas sino que, como defienden Fernández-Enguita, Mena y Riviere (2010), la última palabra la tiene siempre el educando quien es dueño de elegir si aprovechar o no esa segunda oportunidad que se le ofrece. Esta decisión particular se ve, en gran medida, condicionada por modelos de formación atrayentes que sean capaces de conectar con los intereses reales de los más jóvenes, cubriendo sus necesidades básicas sin reportarles el sentimiento de tiempo perdido, de entretenimiento improductivo o de desclasamiento (Bourdieu y Passeron, 2001). Partiendo de esta última circunstancia, se interpreta que reconocerse con una clase social ayuda a los individuos a posicionarse en situaciones más o menos favorables frente a la cultura que es habitualmente legitimada por la Escuela y que reduce, en este sentido, la sensación de desigualdad respecto a la propia conformación social.

Por todo ello, la flexibilidad e individualización, junto a las experiencias previas y la implicación de los empleadores son esenciales (González, 2017). No menos importante es el papel de los educadores en la construcción de tan importante empresa. Tal y como afirman Corchuelo y Cejudo (2015) «es labor de la comunidad educativa y del entorno establecer objetivos reales y asegurarse de que el alumnado conoce a la perfección la vía para conseguirlo (esfuerzo), cómo conseguirlo (aplicando sus capacidades al máximo) y para qué conseguirlo (para acceder a estudios superiores u obtener un título que le abra las puertas al mercado laboral)» (p. 89).

La ausencia de un sistema de sanciones, pese a la existencia de normas, remite un mayor grado de entidad al modelo pedagógico de estas Escuelas, asumiendo que la problemática y características del alumnado son particulares y diversas. Por tanto, la libertad es otro de los fundamentos de las mismas. Libertad para ingresar en las escuelas, para solicitar en cada momento aquellos aprendizajes que estimen más oportunos y adaptados a su situación y para comprometerse con un proyecto de equidad educativa.

En este sentido, los agrupamientos de los escolares se hacen atendiendo no a criterios biológicos, intelectuales o de nivel educativo sino a los intereses del propio alumnado. Así, la homogeneidad a la que antes aludíamos cuando hablábamos de escuelas reactivas queda reducida a la mínima expresión, favoreciendo la construcción de grupos interactivos donde la exclusión social es un riesgo menor. Se sienten, por lo tanto, «parte de» lo que reduce las ganas de rebelarse y abandonar.

Todo este conjunto de propuestas concretas, exclusivas de este tipo de escuelas proactivas, garantizan resultados mucho más efectivos.

\section{PROPUESTAS FUTURAS}

Pese a lo presentado hasta el momento, las E2C siguen siendo una iniciativa poco extendida, aún con el auspicio de la UE. Las escuelas no pueden resolverlo todo (Boal, 2017) y, además, no todos los territorios disponen de estos modelos pedagógicos de atención a la diversidad, lo que supone reducir los beneficios de su intervención a un puñado de «afortunados». Por tanto, las 
políticas socioeducativas futuras deben de ir en la línea de la ampliación, no solo espacial sino también formal, de su marco de acción.

Seguir una senda en la que los valores sociopolíticos sean aprendidos y apr hendidos es vital para trazar una línea recta entre comunidad y Escuela. Asimismo, acabar con la exclusión y con el absentismo se tornan en importantes objetivos que han de perseguir las iniciativas políticas, considerando que la exclusión no es simplemente la falta de sentimiento de pertenencia sino que requiere de una acción y emoción personal en cuanto que miembro activo, participativo de una sociedad. Hablamos, por tanto, de formar auténticos ciudadanos, con derechos y deberes.

La resiliencia es, así mismo, la base sobre la que han de asentarse las propuestas futuras, considerando que solo disponiendo de esa capacidad se podrá hacer frente a los auténticos problemas que va ofreciendo la vida. Tomando en cuenta todo ello, la dificultad para aprender, característica clave de los alumnos de las E2C, se convierte en motivación para el avance en lugar de traba para el aprendizaje.

No menos interesante resulta la acción con el profesorado. Son ellos quienes deben garantizar la formación de estos sujetos activos y son ellos quienes deben de tener establecidas las prioridades pedagógicas de su intervención. Muchas veces, la falta de formación del colectivo docente ofrece trabas a la construcción de estos pequeños grandes hombres y mujeres, por lo que las propuestas futuras han de estar encaminadas a la capacitación excepcional de sus guías.

Como bien se indica en el informe El futuro de la política juvenil del Consejo Europeo: AGENDA 2020, resultado del encuentro internacional en Ucrania en 2008, toda política educativa ha de estar marcada por tres objetivos esenciales:

- Promover la integración de jóvenes excluidos socialmente.

- Garantizar el acceso de los jóvenes a la educación, la formación y la vida laboral, particularmente a través de la promoción y el reconocimiento de su educación no formal y su aprendizaje.

- Apoyar la transición de estos jóvenes del mundo educativo al mercado laboral, ayudándoles, por ejemplo, a que puedan conciliar su vida privada con su empleo (Corchuelo, Cejudo, González-Faraco, Morón, 2016, p.101).

Algunas de las iniciativas que se están poniendo en juego, en relación a ello, tienen lugar en países próximos. Por ejemplo, el país vecino, Portugal, ha comenzado a desarrollar políticas de integración educativa, concediendo a los aprendizajes adquiridos fuera del istema educativo formal, en las Escuelas de Segunda Oportunidad, un valor similar a los que provienen del sistema tradicional. Siendo así, ofrece la posibilidad de otorgar títulos diversos o, en su caso, el reconocimiento de créditos por las competencias adquiridas.

\section{DISCUSIÓN Y CONCLUSIONES}

Tal y como se ha puesto de manifiesto en la presente comunicación, las E2C deben de ser entendidas como centros de formación motivacional y sociocultural, en donde el principal argumento de funcionamiento y finalidad sea la construcción y el empoderamiento de los alumnos.

En este sentido, estamos en disposición de afirmar que estas Escuelas alternativas aportan al panorama europeo un crisol de oportunidades para aquellos colectivos más carenciados, que han sido expulsados del sistema ordinario. Entendemos que la falta de conocimiento y reconocimiento de los distintos sistemas educativos ha dado lugar a la creación de estas Escuelas que desde hace más de dos décadas siguen trabajando por y para las personas.

Las escuelas proactivas, donde se permita atender a cada persona y donde cada una de ellas sea la protagonista de su propio proyecto de vida, se confirman como una buena opción dentro del plantel escolar actual.

Por otro lado, la significación de las personas, el reconocimiento de sus emociones, sentimientos y, por ende, de sus identidades, favorece la emancipación y conversi $n$ en auténticos ciudadanos. Es 
esto lo que pretenden las E2C en aquellos países en las que se han establecido, considerando que aunque se trata de una iniciativa supranacional, solo algunos países, por necesidad o posibilidad, han hecho frente a un articulado más o menos formal de las mismas.

\section{REFERENCIAS BIBLIOGRÁFICAS}

Bonal, X, (2017). ¿Es posible educar en cualquier contexto? Recuperado de http://eldiariodelaeducacion.com/blog/2017/02/16. Bourdieu. P. y Passeron, J. (2001). La reproducción: Elementos para una teoría del sistema de enseñanza. Barcelona: Laia. Comisión Europea (1996). Libro blanco sobre la educación y la formación, Enseñar y aprender. Luxemburgo: Oficina de Publicaciones Oficiales de las Comunidades Europeas.

Corchuelo, C. y Cejudo, C.M. (2013). Atención emocional, motivación y autoestima en el alumnado de las escuelas de segunda de oportunidad: 2-4 de septiembre de 2015 (pp. 83-94). Sevilla: CIECE.

Corchuelo, C., Cejudo, C.M., González-Faraco, J.C., Morón, J.A (2016). Al borde del precipicio: las Escuelas de Segunda Oportunidad, promotoras de inserción social y educativa. International Journal of Educational Research and Innovation (IJERI), 6, 95-109.

Côté, J. y Bynner, J.M. (2008). Changes in the transition to adulthood in the UK and Canada: the role of structure and agency in emerging adulthood. Journal of Youth Studies, 11 (3), 251-268.

Delors, J. (1996). La educación encierra un tesoro. Informe a la UNESCO de la Comisión internacional sobre la educación para el siglo XXI. Madrid: Santillana/UNESCO.

Echeita, G. (2013). Inclusión y exclusión educativa. De nuevo «voz y quebranto». Revista Iberoamericana sobre Calidad, Eficacia y Cambio en Educación, 11(2), 110-118.

European Commission (2018). EPALE. Electronic Platform for Adult Learning inEurope. Recuperado de https://ec.europa.eu/ epale/en/blog/adult-education-centres-germany- between-black-forest-and-europe.

European Commission (2018a). EU Results Examples of EU funded projects. Recuperado de https://ec.europa.eu/budget/ euprojects/second-chance-school_en.

Fernández-Enguita, M., Mena, L. y Riviere, J. (2010). Fracaso y abandono escolar en España. Barcelona. Colección Estudios Sociales, 29. Recuperado de http://smartgroups.es/iesestuaria/wp-content/uploads/2015/06/Fracaso-Escola-_ObraSocialLaCaixa.pdf.

García, M. (2005) Culturas de enseñanza y absentismo escolar en la enseñanza secundaria obligatoria: estudio de casos en la ciudad de Barcelona. Revista de Educación, 338, 347-374.

Gasch y Navas (2017). Expulsados del sistema. Cuadernos de Pedagogía, 478, 1-6.

González, M. T (2017). Desenganche y abandono escolar, y medidas de re-enganche: algunas consideraciones. Profesorado, Revista de currículum y formación del profesorado, 21 (4), 17-37.

González, M. T. y San Fabián, J.L. (2018) Buenas prácticas en medidas y programas para jóvenes desenganchados de lo escolar. REICE. Revista Iberoamericana sobre Calidad, Eficacia y Cambio en Educación, 16(1), 41-60.

Fernández, M. C. (s.f.). La educación inclusiva y las escuelas de segunda oportunidad. Recuperado de http://apoclam. org/archivos-recursos-orientacion/Educacion\%20inclusiva/Experiencias/educacion-inclusiva-y-escuelas-de-segunda-oportunidad.pdf.

Marchesi, A. (2003). El fracaso escolar en España. Madrid: Fundación Alternativas-Documento de trabajo. Recuperado de https://www.nodo50.org/movicaliedu/fracasoescolarespana.pdf.

Olmos, P. y Mas, O. (2013). Jóvenes, fracaso escolar y programas de segunda oportunidad. REOP. 24 (1), 78 - 93.

Prieto, B. (2015). El camino desde la vulnerabilidad escolar hacia el desenganche educativo. El papel de las escuelas de segunda oportunidad en la estrategia contra el abandono educativo. Profesorado. Revista de Currículum y Formación de Profesorado, 19(3),110- 125.

San Fabián; J. L. (1999). La escolaridad obligatoria. Transiciones y tradiciones. Cuadernos de Pedagogía, 282, 25-30.

Tiana, A., Moya J. y Luengo, F. (2011). Implementing Key Competences in Basic Education: reflections on curriculum design and development in Spain. European Journal of Education, 46 (3), 307-322.

Wehmeyer, M. L. (1992). Self-determination and the education of students with mental retardation. Education and Training in Mental Retardation and Developmental Disabilities, 27, 302-314.

Wilkinson, R. y Pickett, K. (2010). The Spirit Level: Why Equality is Better for Everyone. London: Penguin. 